Proceedings of the Edinburgh Mathematical Society (2005) 48, 585-594 (C)

DOI:10.1017/S001309150300049X Printed in the United Kingdom

\title{
NEAR-ISOMETRIES BETWEEN $C(K)$-SPACES
}

\author{
N. J. CUTLAND ${ }^{1 *}$ AND G. B. ZIMMER ${ }^{2}$ \\ ${ }^{1}$ Department of Mathematics, University of Hull, Cottingham Road, \\ Hull HU6 7RX, UK (n.j.cutland@hull.ac.uk) \\ ${ }^{2}$ Department of Computing and Mathematical Sciences, \\ Texas A\&M University-Corpus Christi, 6300 Ocean Drive, \\ Corpus Christi, TX 78412, USA (bzimmer@sci.tamucc.edu)
}

(Received 4 June 2003)

\begin{abstract}
Let $X, Y$ be compact Hausdorff spaces and let $T: C(X, \mathbb{R}) \rightarrow C(Y, \mathbb{R})$ be an invertible linear operator. Non-standard analysis is used to give a new intuitive proof of the Amir-Cambern result that if $\|T\|\left\|T^{-1}\right\|<2$, then there is a homeomorphism $\psi: Y \rightarrow X$. The approach provides a proof of the following representation theorem for such near-isometries:

$$
T f=\left(T 1_{X}\right)(f \circ \psi)+S f,
$$

with $\|S\| \leqslant 2\left(\|T\|-\left(1 /\left\|T^{-1}\right\|\right)\right)$, so $\|S\|<\|T\|$. If $\|T\|\left\|T^{-1}\right\|=1$, then $S=0$, giving the well-known representation for isometries.
\end{abstract}

Keywords: Banach-Stone; non-standard analysis; representation theorem

2000 Mathematics subject classification: Primary 47B38

Secondary $46 \mathrm{~S} 20$

\section{Introduction}

In [4] we gave a new and intuitive proof of the Banach-Stone theorem, which showed how to extend Banach's original proof from the setting of compact metric spaces to compact Hausdorff spaces $X, Y$. For this we used non-standard peak functions: that is, functions of norm 1 in the non-standard extension of $C(X)$ that are supported in the infinitesimal neighbourhood of a single point of $X$. It was shown that a linear isometry $T: C(X) \rightarrow C(Y)$ takes a peak function to a peak function. This induces a bijection between the underlying spaces in a natural way.

In this paper we extend the techniques of $[4]$ to small-norm linear isomorphisms: that is, invertible linear isomorphisms $T: C(X) \rightarrow C(Y)$ such that $\|T\|\left\|T^{-1}\right\|<2$. It was first proved by Amir [1] and Cambern [3] that this still implies that $X$ and $Y$ are homeomorphic. A survey of related results can be found in [6]. Here we prove the AmirCambern result using some new results for peak functions, combined with some ideas

* Present address: Department of Mathematics, University of York, Heslington, York YO10 5DD, UK (nc507@york.ac.uk). 
from Amir's paper. Cambern uses point masses, and showed that the adjoint of $T$ turns a point mass into the orthogonal sum of a point mass and a measure that is of smaller norm. Peak functions do about the same on the function level: we show that a peak function gets mapped to a function that has one distinct peak, but may not necessarily be a peak function. From the proof we obtain a new representation theorem for smallnorm isomorphisms: any such operator $T$ can be written as the sum of a composition operator times $T 1_{X}$ and an operator of smaller norm.

\section{Notation and preliminaries}

A general introduction to non-standard analysis can be found, for example, in [2] or [5]. Any notions and notation that we do not explain can be found in either of those books.

Let $X$ and $Y$ be compact Hausdorff spaces. For a point $x \in X$, the monad of $x$ is defined to be

$$
m(x)=\bigcap\left({ }^{*} U: U \text { is a neighbourhood of } x\right) .
$$

Here ${ }^{*} U$ denotes the non-standard extension of the standard entity $U$.

The first lemma goes back to Robinson [7]. It sums up the properties of the nonstandard extension of a compact Hausdorff space that we need.

Lemma 2.1. Let $X$ be a compact Hausdorff space. Then

(1) for $x, y \in X, m(x) \cap m(y) \neq \emptyset \Longleftrightarrow x=y$;

(2) for each $z \in{ }^{*} X, z \in m(x)$ for some $x \in X$.

By (1) and (2) the standard part map ${ }^{\circ}:{ }^{*} X \rightarrow X$, which assigns the value $x$ to each $z \in m(x)$, is well defined.

We also use another well-known lemma.

Lemma 2.2. If $X, Y$ are compact Hausdorff spaces and $\Phi:{ }^{*} X \rightarrow{ }^{*} Y$ is internal and $S$-continuous (i.e. $x_{1} \approx x_{2}$ implies $\Phi\left(x_{1}\right) \approx \Phi\left(x_{2}\right)$ ), then the (standard) function $\varphi: X \rightarrow Y$ defined by

$$
\varphi(x)={ }^{\circ} \Phi(x) \quad\left(={ }^{\circ} \Phi\left(x^{\prime}\right) \text { for any } x^{\prime} \approx x\right)
$$

is continuous. We write $\varphi={ }^{\circ} \Phi$.

The following lemma about the existence of peak functions is from [4]. Its proof uses Urysohn's lemma.

Lemma 2.3. Let $X$ be a compact Hausdorff space. For each $x \in{ }^{*} X$, there is a nonnegative function $f_{x} \in{ }^{*} C(X)$ with $\left\|f_{x}\right\|_{\infty}=1$, such that the support of $f_{x}$ is contained in $m\left({ }^{\circ} x\right)$ and $f_{x}(x)=1$. This function can be chosen so that the family $\left\{f_{x} x \in{ }^{*} X\right\}$ is internal. 


\section{Definition 2.4.}

(a) A peak function is a non-negative function in ${ }^{*} C(X)$ of norm 1 with support in a single monad.

(b) A peak function $f_{x}$ as given by Lemma 2.3 (with $f_{x}(x)=1$ ) is said to be a peak function for $x$. Figure 1 illustrates such a peak function $f_{x}$.

In this paper we only consider real-valued $C(K)$-spaces. We fix two compact Hausdorff spaces $X, Y$ and write $C(X)$ for $C(X, \mathbb{R})$ and similarly for $Y$. For the rest of the paper we also fix a linear isomorphism

$$
T: C(X) \rightarrow C(Y)
$$

with $\|T\|\left\|T^{-1}\right\|<2$. To help the notation, define

$$
\begin{aligned}
& \alpha=\|T\|, \\
& \beta=\left\|T^{-1}\right\|,
\end{aligned}
$$

so that we have $1 \leqslant \alpha \beta<2$. It is also convenient to write

$$
\delta=\alpha-\frac{1}{\beta} .
$$

Further inequalities that follow from this and are used frequently are

(i) $0 \leqslant \delta=\alpha-\frac{1}{\beta}<\frac{\alpha}{2}<\frac{1}{\beta}$,

(ii) $0 \leqslant \beta-\frac{1}{\alpha}<\frac{\beta}{2}<\frac{1}{\alpha}$;

(iii) note also that for any $f \in C(X)$ and $g \in C(Y)$ we have

$$
\|T f\| \geqslant \frac{1}{\beta}\|f\| \quad \text { and } \quad\left\|T^{-1} g\right\| \geqslant \frac{1}{\alpha}\|g\| .
$$

The following lemma comes from [1] , where Amir used it to define a new operator $\bar{T}$ with $\bar{T} 1_{X}>0$. With our approach we do not need to define a new operator. By using peak functions we can also simplify Amir's lemma.

Lemma 2.5. Let $T: C(X) \rightarrow C(Y)$ be a linear operator with $\alpha \beta<2$. Then

(i) $\left|T 1_{X}(y)\right| \geqslant \alpha(2-\alpha \beta)>0$ for every $y \in Y$;

(ii) $\left|T^{-1} 1_{Y}(x)\right| \geqslant \beta(2-\alpha \beta)>0$ for every $x \in X$.

Proof. (i) Clearly, $\left|T 1_{X}(y)\right| \leqslant \alpha$ for all $y$. Since $\alpha \geqslant \alpha(2-\alpha \beta)$ we need only consider points $y$ with $\left|T 1_{X}(y)\right|<\alpha$.

For any such $y$ take a peak function $g_{y}$; then $\left\|{ }^{*} T^{-1} g_{y}\right\| \geqslant \alpha^{-1}$, so there is $x$ with $\left|{ }^{*} T^{-1} g_{y}(x)\right| \geqslant \alpha^{-1}$. Suppose first that ${ }^{*} T^{-1} g_{y}(x) \geqslant \alpha^{-1}$. 


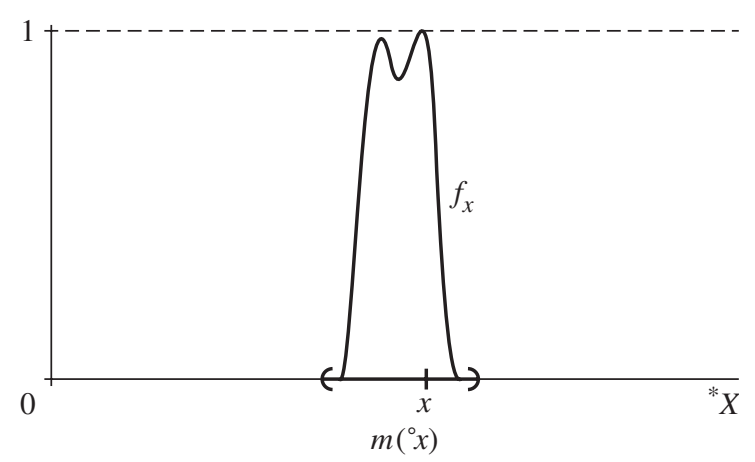

Figure 1. A peak function $f_{x}$ for a point $x \in{ }^{*} X$.

Define $h_{1} \in{ }^{*} C(Y)$ by

$$
h_{1}={ }^{*} T 1_{X}+\left(\alpha-T 1_{X}(y)\right) g_{y} .
$$

We show that $\left\|h_{1}\right\| \approx \alpha$. First we have that $h_{1}(y)=\alpha$, so that $\left\|h_{1}\right\| \geqslant \alpha$. For $y^{\prime} \not \approx y$ we have

$$
\left|h_{1}\left(y^{\prime}\right)\right|=\left.\right|^{*} T 1_{X}\left(y^{\prime}\right) \mid \leqslant \alpha .
$$

For $y^{\prime} \approx y$, since $h_{1} \geqslant{ }^{*} T 1_{X}$ we have $h_{1}\left(y^{\prime}\right) \geqslant-\alpha$. Further, the continuity of $T 1_{X}$ implies that ${ }^{*} T 1_{X}\left(y^{\prime}\right) \approx{ }^{*} T 1_{X}(y)$, so

$$
h_{1}\left(y^{\prime}\right) \approx T 1_{X}(y)+\left(\alpha-T 1_{X}(y)\right) g_{y}\left(y^{\prime}\right) \leqslant T 1_{X}(y)+\alpha-T 1_{X}(y)=\alpha .
$$

This establishes that $\left\|h_{1}\right\| \approx \alpha$.

Then

$$
\begin{aligned}
\alpha \beta \approx\left\|h_{1}\right\| \beta & \geqslant\left\|^{*} T^{-1} h_{1}\right\| \geqslant 1+\left(\alpha-T 1_{X}(y)\right)^{*} T^{-1} g_{y}(x) \\
& \geqslant 1+\left(\alpha-T 1_{X}(y)\right)(1 / \alpha)=2-\frac{T 1_{X}(y)}{\alpha} .
\end{aligned}
$$

This implies that $T 1_{X}(y) \geqslant \alpha(2-\alpha \beta)$.

If ${ }^{*} T^{-1} g_{y}(x) \leqslant-\alpha^{-1}$, then apply the above to $-T$ to obtain $T 1_{X}(y) \leqslant-\alpha(2-\alpha \beta)$.

Part (ii) follows by symmetry.

\section{Corollary 2.6.}

(i) The set $B:=\left\{y \in Y \mid T 1_{X}(y) \geqslant 0\right\}$ is both open and closed and the function $1_{B}$ is continuous.

(ii) The set $A:=\left\{x \in X \mid T^{-1} 1_{Y}(x) \geqslant 0\right\}$ is both open and closed and the function $1_{A}$ is continuous.

Proof. This follows immediately from Lemma 2.5 , since $T 1_{X}$ is continuous and never equal to zero. 


\section{Constructing a homeomorphism $\varphi: X \rightarrow Y$}

In this section we establish that $T$ maps any peak function to a function whose absolute value exceeds $\delta$ in exactly one monad, and that this induces a continuous bijection between $X$ and $Y$.

Lemma 3.1. Let $x \in{ }^{*} X$ with peak function $f_{x}$. Then

(a) if $y \in{ }^{*} B$, then $T f_{x}(y) \geqslant-\delta$;

(b) if $y \notin{ }^{*} B$, then $T f_{x}(y) \leqslant \delta$.

Proof. (a) $\left\|2 f_{x}-1_{X}\right\|=1$ so $\left\|2 T f_{x}-T 1_{x}\right\| \leqslant \alpha$. Hence, for $y \in{ }^{*} B$, we have

$$
\begin{aligned}
2 T f_{x}(y) \geqslant T 1_{x}(y)-\alpha & \geqslant \alpha(2-\alpha \beta)-\alpha \quad(\text { by Lemma 2.5) } \\
& =\alpha(1-\alpha \beta) \geqslant \frac{2}{\beta}(1-\alpha \beta)=-2 \delta .
\end{aligned}
$$

Hence $T f_{x}(y) \geqslant-\delta$, which is part (a).

Part (b) follows by applying (a) to $-T$.

Proposition 3.2. Let $x \in{ }^{*} X$ with peak function $f_{x}$. Then there is a $y_{0} \in{ }^{*} Y$ with

(a) $\left|T f_{x}\left(y_{0}\right)\right| \geqslant(1 / \beta)>\delta ;$

(b) for $y \not \approx y_{0},{ }^{\circ}\left|T f_{x}(y)\right| \leqslant \delta$;

(c) if $y_{0} \in{ }^{*} B$, then $T f_{x}(y) \geqslant-\delta$ for all $y \approx y_{0}$;

(d) if $y_{0} \notin^{*} B$, then $T f_{x}(y) \leqslant \delta$ for all $y \approx y_{0}$.

Proof. (a) Since $\left\|T f_{x}\right\| \geqslant(1 / \beta)$, there is a $y_{0} \in{ }^{*} Y$ with $\left|T f_{x}\left(y_{0}\right)\right| \geqslant(1 / \beta)$. (In fact, from Lemma 3.1, $T f_{x}\left(y_{0}\right) \geqslant(1 / \beta)$ if $y_{0} \in{ }^{*} B$ and $T f_{x}\left(y_{0}\right) \leqslant-(1 / \beta)$ if $y_{0} \notin{ }^{*} B$.)

(b) Take $y_{1} \not \approx y_{0}$ and $g \in C(Y)$ with $\|g\|=1$ and $\left\{{ }^{\circ} g\left(y_{0}\right),{ }^{\circ} g\left(y_{1}\right)\right\}=\{1,-1\}$ and such that ${ }^{*} T^{-1} \bar{g}(x) \geqslant 0$, where the function $\bar{g} \in C(Y)$ is given by

$$
\bar{g}(y)= \begin{cases}g(y) & \text { if } y \in B, \\ -g(y) & \text { if } y \notin B .\end{cases}
$$

Since $\beta \geqslant{ }^{*} T^{-1} \bar{g}\left(x^{\prime}\right) \approx T^{-1} \bar{g}(x) \geqslant 0$ for any $x^{\prime} \approx x$ (and $f_{x}$ is zero elsewhere), we have

$$
\left\|f_{x}-\frac{1}{\beta} T^{-1} \bar{g}\right\| \leqslant 1
$$

and so

$$
{ }^{\circ}\left\|T f_{x}-\frac{1}{\beta} \bar{g}\right\| \leqslant \alpha
$$



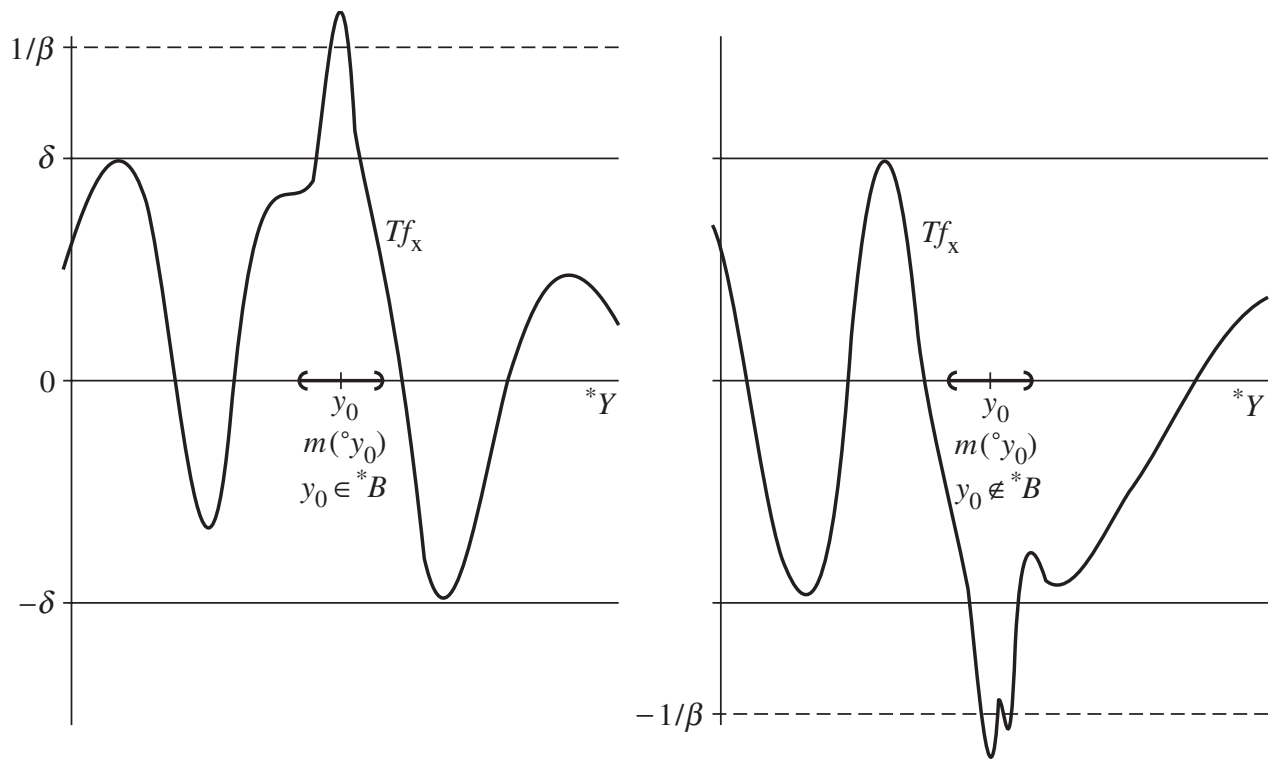

Figure 2. Illustration for Proposition 3.2.

Let $i \in\{0,1\}$ with ${ }^{\circ} g\left(y_{i}\right)=-1$. If $y_{i} \in{ }^{*} B$, then $\bar{g}\left(y_{i}\right)=g\left(y_{i}\right)$ and so

$$
{ }^{\circ} T f_{x}\left(y_{i}\right) \leqslant \alpha+\frac{{ }^{\circ} g\left(y_{i}\right)}{\beta}=\delta,
$$

which means that $i=1$ (since, by (a), we have $T f_{x}\left(y_{0}\right) \geqslant(1 / \beta)>\delta$ if $y_{0} \in{ }^{*} B$ ). This, together with Lemma 3.1 (a), gives ${ }^{\circ}\left|T f_{x}\left(y_{1}\right)\right| \leqslant \delta$, which is (b) in this case.

If $y_{i} \notin^{*} B$, then $\bar{g}\left(y_{i}\right)=-g\left(y_{i}\right)$ and so

$$
{ }^{\circ} T f_{x}\left(y_{i}\right) \geqslant-\alpha-\frac{{ }^{\circ} g\left(y_{i}\right)}{\beta}=-\delta,
$$

so that again $i=1$. So ${ }^{\circ} T f_{x}\left(y_{1}\right) \geqslant-\delta$, giving ${ }^{\circ}\left|T f_{x}\left(y_{1}\right)\right| \leqslant \delta$ when combined with Lemma $3.1(\mathrm{a})$.

For (c) and (d), since $B$ is both open and closed, for $y \approx y_{0}$ we have $y \in{ }^{*} B$ if and only if $y_{0} \in{ }^{*} B$. Now use Lemma 3.1 .

Note that Proposition 3.2 can be summarized as follows (see Figure 2). Given any peak function $f_{x}$ for $x \in{ }^{*} X$, its image ${ }^{*} T f_{x}$ has standard part bounded by $\pm \delta$ except on a single monad, where it peaks at some point $y_{0}$ with value $\left|T f_{x}\left(y_{0}\right)\right| \geqslant(1 / \beta)>\delta$. For all $y \approx y_{0}$ its values only go outside the interval $[-\delta, \delta]$ in one direction (depending on whether or not $\left.y_{0} \in{ }^{*} B\right)$.

Definition 3.3. Let $f_{x} \in{ }^{*} C(X)$ be a peak function. We say that ${ }^{*} T f_{x}$ peaks at $y \in{ }^{*} Y$ if $\left|{ }^{*} T f_{x}(y)\right| \geqslant(1 / \beta)$. Similarly, we say for a peak function $g_{y} \in{ }^{*} C(Y)$ that ${ }^{*} T^{-1} g_{y}$ peaks at $x \in{ }^{*} X$ if $\left|{ }^{*} T^{-1} g(x)\right| \geqslant(1 / \alpha)$. 
Note that $\left\|{ }^{*} T f_{x}\right\| \geqslant(1 / \beta)>\delta$. So Proposition 3.2 tells us that for each peak function $f_{x} \in{ }^{*} C(X),{ }^{*} T f_{x}$ peaks in exactly one monad and on that monad the peak is above $\delta$ if the monad is contained in ${ }^{*} B$ and below $-\delta$ if the monad is contained in ${ }^{*}(Y \backslash B)$. Outside that monad, ${ }^{\circ}{ }^{*} T f_{x}(y) \mid \leqslant \alpha-(1 / \beta)$.

A modification of the proof of Proposition 3.2 (b) yields the following corollary.

Corollary 3.4. Let $x_{1}, x_{2} \in{ }^{*} X, x_{1} \approx x_{2}$ with peak functions $f_{x_{1}}$ and $f_{x_{2}}$, respectively. Then ${ }^{*} T f_{x_{1}}$ and ${ }^{*} T f_{x_{2}}$ peak in the same monad.

Proof. Assume that ${ }^{*} T f_{x_{1}}$ and ${ }^{*} T f_{x_{2}}$ peak in different monads. Then there are points $y_{1}, y_{2} \in{ }^{*} Y$ with $y_{1} \not \approx y_{2}$ such that $\left|{ }^{*} T f_{x_{i}}\left(y_{i}\right)\right| \geqslant(1 / \beta)$ for $i=1,2$. By Urysohn's lemma there exists a standard $g \in C(Y)$ with $\|g\|=1$ such that $g\left(y_{1}\right) \approx 1$ and $g\left(y_{2}\right) \approx-1$. Without loss of generality we may assume that ${ }^{\circ *} T^{-1} \bar{g}\left(x_{1}\right)={ }^{\circ *} T^{-1} \bar{g}\left(x_{2}\right) \geqslant 0$ (otherwise replace $g$ with $-g$ and relabel $y_{1}$ and $y_{2}$ ).

Arguing as in the previous proof we have

$$
\left\|f_{x_{2}}-\frac{1}{\beta} T^{-1} \bar{g}\right\| \leqslant 1
$$

and so

$$
\left\|T f_{x_{2}}-\frac{1}{\beta} \bar{g}\right\| \leqslant \alpha
$$

If $y_{2} \in{ }^{*} B$, then $\bar{g}\left(y_{2}\right)=g\left(y_{2}\right)$ and so

$$
{ }^{\circ} T f_{x_{2}}\left(y_{2}\right) \leqslant \alpha+\frac{{ }^{\circ} g\left(y_{2}\right)}{\beta}=\delta,
$$

which contradicts the hypothesis that $T f_{x_{2}}$ peaks at $y_{2}$.

Similarly, if $y_{2} \notin{ }^{*} B$, then $\bar{g}\left(y_{2}\right)=-g\left(y_{2}\right)$ and so

$$
{ }^{\circ} T f_{x_{2}}\left(y_{2}\right) \geqslant-\alpha-\frac{{ }^{\circ} g\left(y_{2}\right)}{\beta}=-\delta,
$$

again contradicting the hypothesis.

The above two results (Proposition 3.2 and Corollary 3.4) allow the definition of an S-continuous (internal) mapping $\Phi:{ }^{*} X \rightarrow{ }^{*} Y$ as follows. Let $\left\{f_{x}\right\}_{x \in{ }^{*} X}$ be an internal family of peak functions and define

$$
\Phi(x)=\text { a point in }{ }^{*} Y \text { where }{ }^{*} T f_{x} \text { peaks }
$$

(i.e. $\Phi(x)$ is a point where $\left\|^{*} T f_{x}\right\|$ assumes its maximum). Similarly, we may define an S-continuous internal mapping $\Psi:{ }^{*} Y \rightarrow{ }^{*} X$ using an internal family $\left\{g_{y}\right\}_{y \in *^{*}}$ of peak functions.

The next crucial result shows that $\Phi$ and $\Psi$ are essentially inverse to one another, and will be used to show that $X$ and $Y$ are homeomorphic. 
Proposition 3.5. Let $x \in{ }^{*} X$ with peak function $f_{x}$. If ${ }^{*} T f_{x}$ peaks at $y \in{ }^{*} Y$, and $g_{y}$ is any peak function for $y$, then ${ }^{*} T^{-1} g_{y}$ peaks in the monad of $x$.

Proof. Let $f_{x}$ be a peak function for $x \in{ }^{*} X$ with ${ }^{*} T f_{x}$ peaking at $y$. That is, $\left|T f_{x}(y)\right| \geqslant(1 / \beta)$ and ${ }^{\circ}\left|T f_{x}\left(y^{\prime}\right)\right| \leqslant \delta$ for $y^{\prime} \not \approx y$. By Corollary 3.4, any two peak functions for $y$ have their images under ${ }^{*} T^{-1}$ peaking in the same monad, so it is sufficient to prove the result for one particular peak function $g_{y}$, which we now define.

Assume first that $y \in B$. We split off the part of ${ }^{*} T f_{x}$ that peaks above $\delta$ and normalize it to a peak function $g_{y}$. Explicitly, set

$$
g_{y}\left(y^{\prime}\right)=\max \left(\frac{* T f_{x}\left(y^{\prime}\right)-\delta}{\left\|T f_{x}\right\|-\delta}, 0\right)
$$

We will show that

$$
\left\|^{*} T f_{x}-\alpha g_{y}\right\| \leqslant \delta=\alpha-\frac{1}{\beta} .
$$

Once this is proved we have $\left|f_{x}-\alpha^{*} T^{-1} g_{y}\right| \leqslant \beta \alpha-1$, and so, for $x^{\prime} \not x$,

$$
\left|T^{* 1} g_{y}\left(x^{\prime}\right)\right| \leqslant \beta-\frac{1}{\alpha} .
$$

From Proposition 3.2 (applied to $\left.T^{-1}: C(Y) \rightarrow C(X)\right)$ this means that $T^{-1} g_{y}$ peaks in the monad of $x$.

To check (3.1), if $\left|T f_{x}\left(y^{\prime}\right)\right| \leqslant \delta$, then $g_{y}\left(y^{\prime}\right)=0$ so $\left|T f_{x}\left(y^{\prime}\right)-\alpha g_{y}\left(y^{\prime}\right)\right| \leqslant \delta$. If $T f_{x}\left(y^{\prime}\right)>$ $\delta$, then

$$
T f_{x}\left(y^{\prime}\right)-\alpha g_{y}\left(y^{\prime}\right)=\delta+\left(\left\|{ }^{*} T f_{x}\right\|-\delta-\alpha\right) g_{y}\left(y^{\prime}\right) .
$$

Result (3.1) follows from observing that

$$
0 \leqslant \delta+\alpha-\left\|^{*} T f_{x}\right\| \leqslant \delta+\alpha-\frac{1}{\beta}=2 \delta .
$$

If $y \notin B$, then apply what we have already proved to the operator $-T$.

We now have all the ingredients for our proof of the following.

Theorem 3.6 (Amir, Cambern). Let $X$ and $Y$ be compact Hausdorff spaces. If there is an invertible linear operator $T: C(X) \rightarrow C(Y)$ with $\|T\|\left\|T^{-1}\right\|<2$, then $X$ and $Y$ are homeomorphic.

Proof. Take the internal S-continuous functions $\Phi:{ }^{*} X \rightarrow{ }^{*} Y$ and $\Psi:{ }^{*} Y \rightarrow{ }^{*} X$ defined above. From these, define continuous mappings $\varphi={ }^{\circ} \Phi: X \rightarrow Y$ and $\psi=$ ${ }^{\circ} \Psi: Y \rightarrow X$ using Lemma 2.2. Proposition 3.5, together with Corollary 3.4, tells us that $\Psi(\Phi(x)) \approx x$ for all $x \in{ }^{*} X$, and similarly $\Phi(\Psi(y)) \approx y$ for all $y \in{ }^{*} Y$. Hence $\psi=\varphi^{-1}$. 


\section{Representation of small-norm isomorphisms}

It is well known that all isometries between $C(K)$-spaces are (up to a sign function) composition operators of the form $(T f)(y)=g(y) f(\psi(y))$, where $g=T 1_{X} \in C(Y)$ with $|g(y)|=1$ for all $y \in Y$ and $\psi: Y \rightarrow X$ is a homeomorphism. In this section we generalize this result to near-isometries: every near-isometry is the sum of a composition operator times the image of the function constant 1 and an operator of smaller norm.

Theorem 4.1. Let $T: C(X) \rightarrow C(Y)$ be an invertible linear operator such that $\alpha \beta<2$. Then there is a homeomorphism $\psi: Y \rightarrow X$, a function $g=T 1_{X} \in C(Y)$ with $\|g\| \leqslant \alpha$ and a linear operator $S: C(X) \rightarrow C(Y)$ with

$$
\|S\| \leqslant 2\left(\alpha-\frac{1}{\beta}\right)<\alpha
$$

such that

$$
(T f)(y)=g(y) f(\psi(y))+(S f)(y) \quad \text { for all } y \in Y .
$$

Proof. Let $\psi: Y \rightarrow X$ be the homeomorphism given by Theorem 3.6. Take $f \in C(X)$ with $f \geqslant 0$ and $\|f\|=1$. Fix a $y \in Y$ and consider the function $f-f(\psi(y)) \in C(X)$. Take a peak function $f_{\psi(y)} \in{ }^{*} C(X)$ for the point $\psi(y)$. For $x \approx \psi(y),{ }^{*} f(x) \approx f(\psi(y))$. For $x \notin m(\psi(y)), f_{\psi(y)}(x) \approx 0$. Hence

$$
{ }^{\circ}\left\|f-f(\psi(y)) \pm f_{\psi(y)}\right\|=1
$$

and therefore $\left\|T f-f(\psi(y))\left(T 1_{X}\right) \pm T f_{\psi(y)}\right\| \leqslant \alpha$. By construction of $\psi$ we know that $\left|T f_{\psi(y)}(\bar{y})\right| \geqslant(1 / \beta)$ for some point $\bar{y} \approx y$. Hence

$$
\left|(T f)(\bar{y})-f(\psi(y))\left(T 1_{X}\right)(\bar{y})\right| \leqslant \alpha-\frac{1}{\beta} .
$$

Since $T f-f(\psi(y)) T 1_{X}$ is a standard function and $\bar{y} \approx y$, this implies that

$$
\left|(T f)(y)-f(\psi(y))\left(T 1_{X}\right)(y)\right| \leqslant \alpha-\frac{1}{\beta} .
$$

Define an operator $S: C(X) \rightarrow C(Y)$ by

$$
(S f)(y)=(T f)(y)-f(\psi(y))\left(T 1_{X}\right)(y) .
$$

For non-negative functions $f$ we showed that

$$
\|S f\| \leqslant\|f\|\left(\alpha-\frac{1}{\beta}\right) .
$$

An analogous argument works for non-positive functions $f \in C(X)$. Decomposing any $f \in C(X)$ into $f^{+}$and $f^{-}$finishes the proof, since

$$
\|S f\| \leqslant\left\|T f^{+}-\left(f^{+} \circ \psi\right)\left(T 1_{X}\right)\right\|+\left\|T f^{-}-\left(f^{-} \circ \psi\right)\left(T 1_{X}\right)\right\| \leqslant 2\|f\|\left(\alpha-\frac{1}{\beta}\right) .
$$


Corollary 4.2. Any bijective linear isometry $T: C(X) \rightarrow C(Y)$ is of the form

$$
T f(y)=\left(T 1_{X}\right)(y) f(\psi(y)),
$$

where $\psi: Y \rightarrow X$ is a homeomorphism.

Proof. The proof follows immediately from Theorem 4.1, since here

$$
\|S\|=2\left(\alpha-\frac{1}{\beta}\right)=0 .
$$

\section{The complex case}

Amir's proof [1] of the Amir-Cambern theorem was for real-valued functions only, whereas Cambern's proof in [3] is for complex-valued functions. A natural question is whether the ideas we have developed in this paper to prove the real-valued version extend to the complex case. Here we have to report complete lack of success. The infinitesimal approach above draws on ideas in Amir's paper - we have been unable to find a natural way to generalize these. A first goal in this direction would be to handle the complex isometric case: by finding a characterization of complex peak functions that is invariant under isometries, as in [4]. Even here, unfortunately, there has been no progress to date.

Acknowledgements. The work of G.B.Z. was supported by a Faculty Research Grant from Mississippi University for Women.

\section{References}

1. D. Amir, On isomorphisms of continuous function spaces, Israel J. Math. 3 (1966), 205210.

2. L. O. Arkeryd, N. J. Cutland and C. W. Henson (eds), Nonstandard analysis, theory and applications, NATO ASI Series (Dordrecht, Kluwer, 1997).

3. M. Cambern, A generalized Banach-Stone theorem, Proc. Am. Math. Soc. 17 (1966), 396-400.

4. N. J. Cutland and G. B. Zimmer, A new proof of the Banach-Stone theorem, Bull. Aust. Math. Soc. 57 (1998), 55-58.

5. A. HuRd AND P. A. Loeb, An introduction to nonstandard real analysis (Academic Press, 1985).

6. K. JAROSZ, Isometries and small bound isomorphisms of function spaces, in Function Spaces, Edwardsville, IL, 1990, Lecture Notes in Pure and Applied Mathematics, vol. 136 (Dekker, New York, 1992).

7. A. Robinson, Non-standard analysis, 2nd edn (North-Holland, Amsterdam, 1974). 